\title{
A Further Investigation of the Recharge Oscillator Paradigm for ENSO Using a Simple Coupled Model with the Zonal Mean and Eddy Separated
}

\author{
SOON-IL AN* AND IN-SIK KANG \\ Department of Atmospheric Sciences, Seoul National University, Seoul, South Korea
}

15 June 1998 and 22 October 1999

\begin{abstract}
The recharge oscillator paradigm for ENSO is further investigated by using a simple coupled model, which externally includes the equatorial wave dynamics represented by the Kelvin and gravest symmetric Rossby waves. To investigate the role of eddies in the Pacific basin-wide adjustment to the wind forcing, particularly at the western and eastern boundaries, the zonal mean and eddy parts are treated separately in the current model. It is clearly demonstrated that the basin-wide adjustment of the tropical ocean is accomplished by the net mass transport induced by the meridional transport over the tropical ocean interior and the zonal fluxes at the boundaries. With a reasonable choice of the reflection coefficient, particularly at the western boundary, the meridional transport plays a bigger role than the zonal boundary flux and determines the sign of zonal-mean thermocline depth tendency, in a way that the discharge of equatorial mass in the warm phase and recharge in the cold phase serve as a phase transition mechanism of the coupled system. The meridional mass transport is induced mainly by a geostrophic current associated with the east-west slope of thermocline depth, established quickly by the wind forcing. Also discussed in this paper is the difference between the recharge oscillator and the delayed oscillator in explaining the phase transition mechanism of ENSO.
\end{abstract}

\section{Introduction}

Since Bjerknes (1969), a number of studies have investigated and proposed the dynamics of the El NiñoSouthern Oscillation (ENSO; Philander et al. 1984; Wyrtki 1985; Zebiak and Cane 1987; Hirst 1988; Suarez and Shopf 1988; Battisti and Hirst 1989; Jin 1997a; and others). It is now widely accepted that the ENSO is an oscillation phenomenon repeated by adjustment processes of the coupled ocean-atmosphere system in the tropical Pacific. The adjustment processes examined extensively thus far are the equatorial wave propagations with a time delay (Battisti and Hirst 1989; Suarez and Schopf 1988) and the basin-wide heat and mass exchange between the equator and off-equator (Wyrtki 1985; Jin 1997a,b; Li 1997). In the present study, the basin-wide adjustment process [called the recharge oscillator mechanism in Jin 1997a,b)] is more intensively investigated by using a simple coupled system with the zonal mean and eddy parts separated.

\footnotetext{
* Current affiliation: International Pacific Research Center, SOEST, University of Hawaii, Honolulu, Hawaii.

Corresponding author address: Prof. In-Sik Kang, Department of Atmospheric Sciences, Seoul National University, Seoul, 151-742 South Korea.

E-mail: kang@climate.snu.ac.kr
}

In an earlier work, Zebiak and Cane (1987) demonstrated the importance of basin-wide heat content in ENSO by showing that the interannual oscillation could not be generated by removing the effect of zonal-mean part of thermocline depth anomaly in the SST tendency equation. Schneider et al. (1995), in their the ocean model experiments, showed that the eddy part (defined as the deviation from the zonal mean) of the heat content was approximately balanced by the wind stress, but the zonal mean (hereafter defined as an area average over the Pacific basin) is in nonequilibrium with the wind stress and provides the oscillation memory of the coupled system. The role of equatorial zonal-mean heat content in the interannual oscillation of the coupled system is more generally explained by the so-called recharge oscillator theory of Jin (1997a,b). He suggested that the adjustment takes place by means of Sverdrup transport, either pumping the mass in or out of the equatorial region depending on wind direction.

The recharge-discharge mechanism of Jin (1997a) is plausible. But, in considering the basin-wide adjustment, Jin's model casts the SST anomaly over the eastern part of the equatorial Pacific and the thermocline depth over the warm pool region of the basin. Wang and Fang (1996) also obtained the oscillatory solution in terms of the local thermocline depth and SST both in the eastern Pacific. Both studies consider the variation of basin-wide heat content using a local variation of thermocline depth either in the western Pacific or in the 
eastern Pacific rather than using the zonally integrated statistics. On the other hand, Li (1997) examined the ENSO cycle using a zonal-mean simple coupled model. But Li (1997) did not consider the role of boundary reflection. However, as shown by the present study and Jin (1997b), the boundary reflection is an important factor for the phase transition of the ENSO cycle. In the present study, in particular, by using the linearized shallow water system with the zonal mean and eddy separated, the zonal mean and eddy interaction is explicitly considered at the boundaries.

Section 2 explains the formulation of the present coupled model. The unstable mode of the present model mimicking ENSO is obtained in section 3, and the zonal mean and eddy parts of the unstable oscillatory mode are examined. The transition mechanism of ENSO is discussed in section 4. Conclusions are given in section 5.

\section{Model}

In the present study, a linear shallow water equation is used for the ocean model with a long wave approximation in an equatorial beta plane, and the thermodynamic equation for sea surface temperature (SST) has a linearized zonal advection and upwelling terms (Hirst 1988). The model is further simplified by truncating higher meridional wave modes other than the Kelvin and gravest symmetric Rossby modes. The present model with the two wave components is the same as that of Kang and An (1998). Now the Kang and An equations are divided into two sets of equations governing the zonal mean and the deviation (eddy) from the zonal mean as follows:

$$
\begin{aligned}
\frac{\partial\left[S_{0}\right]}{\partial t}+\frac{S_{0}\left(x_{E}\right)-S_{0}\left(x_{W}\right)}{L}-\left[\tau_{0}\right] & =-a\left[S_{0}\right], \\
\frac{\partial\left[S_{2}\right]}{\partial t}-\frac{S_{2}\left(x_{E}\right)-S_{2}\left(x_{W}\right)}{3 L}+\frac{\sqrt{2}}{3}\left[\tau_{0}\right] & =-a\left[S_{2}\right], \\
\frac{\partial\left[T_{0}\right]}{\partial t}-\alpha\left[S_{0}\right]-\beta\left[S_{2}\right] & =-d\left[T_{0}\right], \\
\frac{\partial S_{0}^{*}}{\partial t}+\frac{\partial S_{0}^{*}}{\partial x}-\frac{S_{0}\left(x_{E}\right)-S_{0}\left(x_{W}\right)}{L}-\tau_{0}^{*} & =-a S_{0}^{*}, \\
\frac{\partial S_{2}^{*}}{\partial t}-\frac{1}{3} \frac{\partial S_{2}^{*}}{\partial x}+\frac{S_{2}\left(x_{E}\right)-S_{2}\left(x_{W}\right)}{3 L}+\frac{\sqrt{2}}{3} \tau_{0}^{*} & =-a S_{2}^{*}, \\
\frac{\partial T_{0}^{*}}{\partial t}-\alpha S_{0}^{*}-\beta S_{2}^{*} & =-d T_{0}^{*},
\end{aligned}
$$

where $S=h+u$ and $R=h-u ; h$ and $u$ are the thermocline depth and zonal current, respectively; $T$ is the $\mathrm{SST} ; \tau$ is the wind stress; and $a$ and $d$ are the Rayleigh friction and Newtonian cooling coefficients, respectively. The square bracket and superscript star indicate the zonal mean and the deviation, respectively, and the subscripts 0 and 2 indicate the Kelvin and gravest Rossby components, respectively. Further, $\alpha=\frac{1}{2}\left[-\bar{T}_{x}+\int K_{T}(y) \psi_{0} \psi_{0} d y\right], \beta=$ $(\sqrt{2} / 2)\left[\bar{T}_{x}+\int K_{T}(y) \psi_{0}\left(\psi_{2} / \sqrt{2}+\psi_{0}\right) d y\right]$, and $\psi_{0}$ and $\psi_{2}$ are the zeroth and second order Hermite polynomials, respectively. Subscripts $E$ and $W$ indicate the eastern and western boundaries, respectively, and $L$ is the length scale of the ocean basin. Other notations are conventional and the values of coefficients used are same as those of Kang and An (1998). Equations (1)-(3) and Eqs. (4)-(6) govern the zonal mean and eddy parts of the coupled system, respectively.

The eddy part of the coupled system including Eqs. (4)-(6) is apparently a closed equation set having the eddy term only. However, the eddy equations cannot be solved without the zonal means, because the zonal mean and eddy parts are communicating through the boundaries. In the model, the reflection at both boundaries is expressed such as $S_{0}\left(x_{W}\right)=r_{W} S_{2}\left(x_{W}\right)$ and $S_{2}\left(x_{E}\right)=$ $r_{E} S_{0}\left(x_{E}\right)$, where $r_{W}$ and $r_{E}$ are the reflection coefficient at the western and eastern boundaries, respectively. From the above relationship, the boundary values for the eddy can be written as $S_{0}^{*}\left(x_{W}\right)=-\left[S_{0}\right]+r_{W}\left[S_{2}\right]$ $+r_{W} S_{2}^{*}\left(x_{W}\right)$ and $S_{2}^{*}\left(x_{E}\right)=-\left[S_{2}\right]+r_{E}\left[S_{0}\right]+r_{E} S_{0}^{*}\left(x_{E}\right)$. Thus, the reflection cannot be expressed by the eddy alone. The value of both reflection coefficients used is $1 /(2)^{1 / 2}$.

\section{Time evolution of the coupled unstable mode}

The present model has two unstable modes: A nonoscillating unstable mode and an unstable oscillatory mode. The nonoscillating unstable mode has no eddy structure but only zonal means. However, in reality the zonal mean and eddy interact with each other. Thus, the first unstable mode is unrealistic. In this section, the evolution feature of the second unstable mode is examined. The period of oscillation and the $e$-folding time of this mode are 2.5 and $3.7 \mathrm{yr}$, respectively. All results in this study are based on this unstable eigenmode, and its time variation is examined in terms of the real part of the mode. Note that this oscillating unstable mode is the same as the ENSO mode described in Kang and An (1998), where they obtained the eigenmode without separating the zonal mean and eddy.

Figure 1 shows a half-cycle of the variations of SST and the thermocline depth of the oscillating unstable mode. The variations are plotted with a $45^{\circ}$ phase interval. Hereafter, the mature phase of warm SST is represented by the phase of $0^{\circ}$. The SST variation shown in Figs. 1a-e, is characterized mainly by a standing oscillation over the central and eastern Pacific, although there is little eastward propagation signal. In contrast, the thermocline variation is relatively large in the western Pacific. In a quarter-cycle before the mature phase of SST (Fig. 1f), when SST is very weak, a positive thermocline depth anomaly appears in the western tropical Pacific. After the transition phase (Figs. 1g-j), the thermocline depth variation is characterized by an eastwest seesaw pattern. These evolution features of SST 


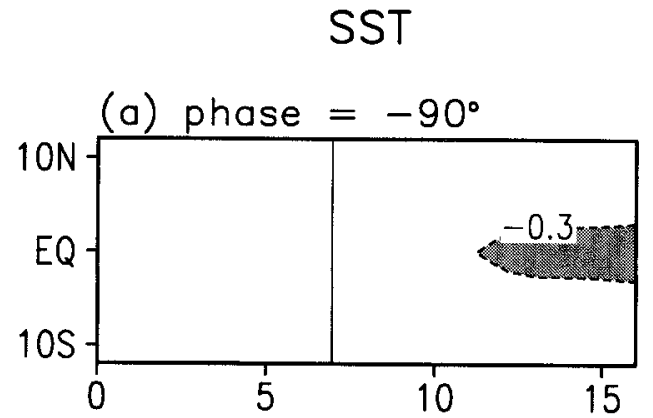

Thermocline depth

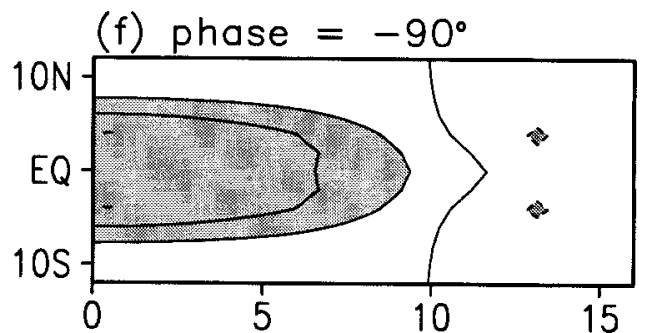

(b) $-45^{\circ}$

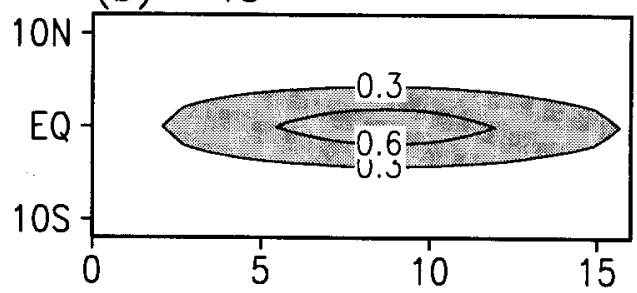

(c) $0^{\circ}$

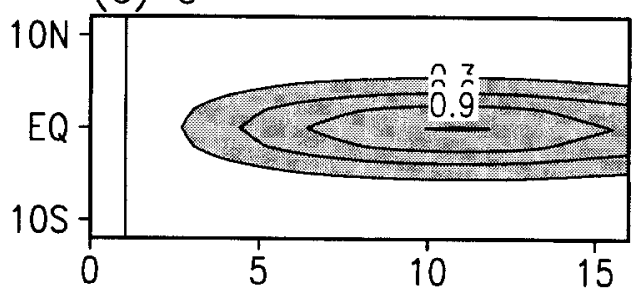

(d) $+45^{\circ}$

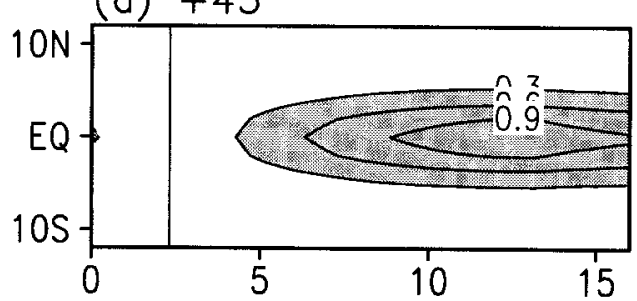

(e) $+90^{\circ}$

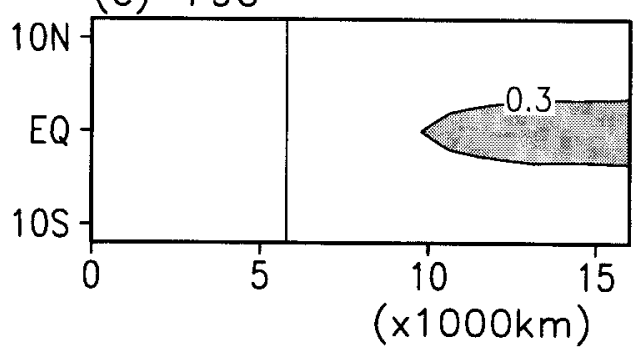

(g) $-45^{\circ}$

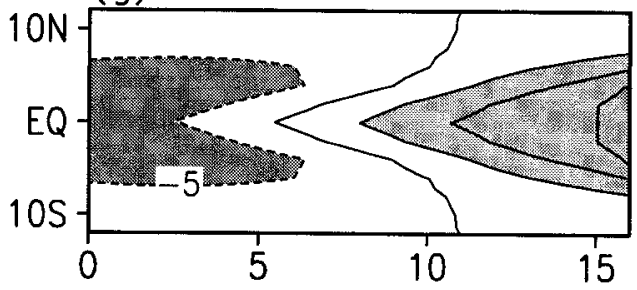

(h) $0^{\circ}$
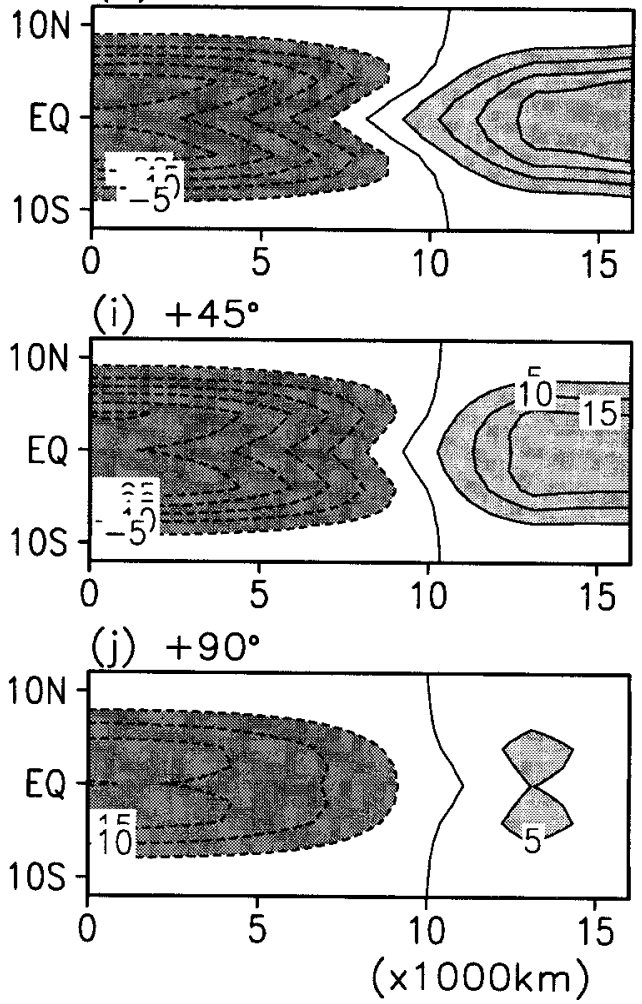

FIG. 1. Variations of SST $\left({ }^{\circ} \mathrm{C}\right)$ and thermocline depth $(\mathrm{m})$ of the unstable oscillating mode for a half-cycle of the oscillation. Those are plotted with a $45^{\circ}$ phase interval centered at the mature phase of warm SST. Negative values are shaded.

and thermocline depth are consistent with the results of the ocean-atmosphere coupled general circulation model (Chao and Philander 1993), indicating that the present truncated system depicts the essential feature of ENSO.

The relationship between the zonal-mean thermocline depth and SST in the equator is shown in Fig. 2. It is very clear in the figure that the zonal-mean thermocline depth leads the SST by a quarter-cycle. On the other hand, the eddy part of thermocline depth varies in phase with SST (Fig. 2). In particular, the east-west thermocline slope $\left(h_{E}-h_{W}\right)$ has an almost simultaneous relationship with the zonal mean SST, indicating that the 


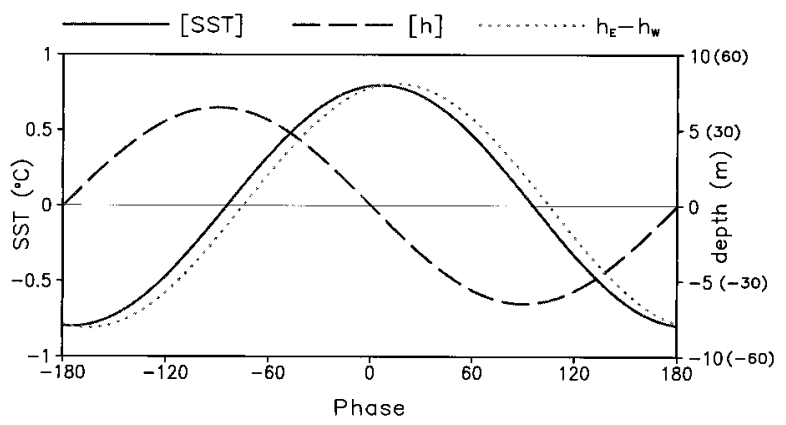

FIG. 2. Variations of the zonal-mean SST, the zonal-mean thermocline depth, and the thermocline slope $\left(h_{E}-h_{W}\right)$ at the equator for one cycle. The scales of the zonal-mean thermocline depth and the thermocline slope are indicated with and without the parentheses of the right abscissa.

east-west slope is quickly adjusted to the SST and wind stress forcing.

\section{Phase transition mechanism}

To understand the transition mechanism of the coupled system, the equation governing the zonal-mean thermocline depth, in which the memory of the coupled system resides, is examined. The equation can be written as

$$
\frac{\partial[h]}{\partial t}=-\frac{u_{E}-u_{W}}{L}-\frac{\partial[v]}{\partial y}-a[h] .
$$

It indicates that the $[h]$ tendency results from the boundary flux $\left(u_{W}-u_{E}\right) / L$ as well as the meridional divergence. Generally the normal component of the current along the boundaries, for example, $u_{W}$ and $u_{E}$, should vanish. Under the long-wave approximation that excludes short Rossby waves, however, $u_{W}$ does not vanish, but the meridional integral of $u_{W}$ is zero (Cane and Sarachik 1981). The variations of the two terms at the equator, the boundary flux and meridional divergence terms, are shown in Fig. 3. As shown in the figure, the two terms have opposite signs to each other, and the absolute magnitude of meridional momentum convergence $(-\partial[v] / \partial y)$ is always bigger than that of the boundary flux term. Therefore the tendency of the zonalmean thermocline depth is determined by the cancellation between the two terms and controlled mainly by the meridional divergence term. It is noted that since the meridional divergence is proportional to SST (shown below), the $[h]$ tendency is proportional to the SST with an opposite sign, and thus $[h]$ and $[\mathrm{SST}]$ have a quadrature phase relationship.

Now we discuss the mechanisms controlling both the meridional current and boundary fluxes. In the present truncated model, the meridional current can be expressed as

$$
\left[v_{1}\right]=\frac{2}{3} \frac{S_{2}\left(x_{E}\right)-S_{2}\left(x_{W}\right)}{L}-\frac{\sqrt{2}}{6} \mu\left[T_{0}\right],
$$

where $\left[v_{1}\right]$ is the amplitude of meridional current associated with the first asymmetric Hermite function. Equation (8) is easily obtained from the same equations that were used in the derivation of Eq. (2), by eliminating $\left[S_{2}\right]$ rather than $\left[v_{1}\right]$. The first term on the righthand side of Eq. (8) expresses the meridional current associated with the zonal slope of thermocline depth, and the second term the Ekman transport. In particular, the first term can be written as $\int\left\{\left(h_{E}-h_{W}\right) / L\right\} d y$, since $\int u_{E} d y$ and $\int u_{W} d y$ are zero (Hirst 1988), and thus this term represents the geostrophic current induced by the mean east-west thermocline slope along the meridional direction. The two terms in Eq. (8) have opposite signs to each other (not shown here). During a warm phase, westerly wind stress near the equator produces the geostrophic current toward the pole through inducing the eastward thermocline slope, while the westerly wind produces the Ekman transport toward the equator. The geostrophic current in the present model is about four times bigger than the Ekman current (not shown), and thus $\left[v_{1}\right]$ is determined mainly by the geostrophic current induced by the wind stress without time delay. Note that the positive (negative) value of $\left[v_{1}\right]$ results in the meridional divergence (convergence) at the equator, because of the meridional structure of Hermite function. Therefore, the meridional divergence, resulting in the $[h]$ tendency in Eq. (7), is proportional to the wind stress and SST.

In the following, we examine the sensitivity of the terms in Eq. (7) to the boundary reflection. By separating the meridional mode and after neglecting the damping term, Eq. (7) can be written as

$$
\begin{aligned}
\frac{\partial[h]}{\partial t} & =\left(B_{0}-M_{0}\right) \Psi_{0}+\left(B_{2}-M_{2}\right) \Psi_{2}, \\
B_{0} & =\int \frac{u_{W}-u_{E}}{L} \Psi_{0} d y \\
& =\frac{1}{2 L}\left\{\left(r_{W}-\sqrt{2}\right) S_{2}\left(x_{W}\right)\right. \\
M_{0} & =\int \frac{\partial\left[v_{1}\right]}{\partial y} \Psi_{0} d y \\
& =\frac{\sqrt{2}}{3} \frac{r_{E} S_{0}\left(x_{E}\right)-S_{2}\left(x_{W}\right)}{L}-\frac{1}{6}\left[\tau_{0}\right], \\
B_{2} & =\int \frac{\left.u_{W}-S_{0}\left(x_{E}\right)\right\},}{L} \Psi_{2} d y \\
& =-\frac{1}{2 L}\left\{r_{E} S_{0}\left(x_{E}\right)-S_{2}\left(x_{W}\right)\right\}, \\
& =-\left\{\frac{2}{3} \frac{r_{E} S_{0}\left(x_{E}\right)-S_{2}\left(x_{W}\right)}{L}-\frac{\sqrt{2}}{6}\left[\tau_{0}\right]\right\}, \\
M_{2} & =\int \frac{\partial\left[v_{1}\right]}{\partial y} \Psi_{2} d y
\end{aligned}
$$




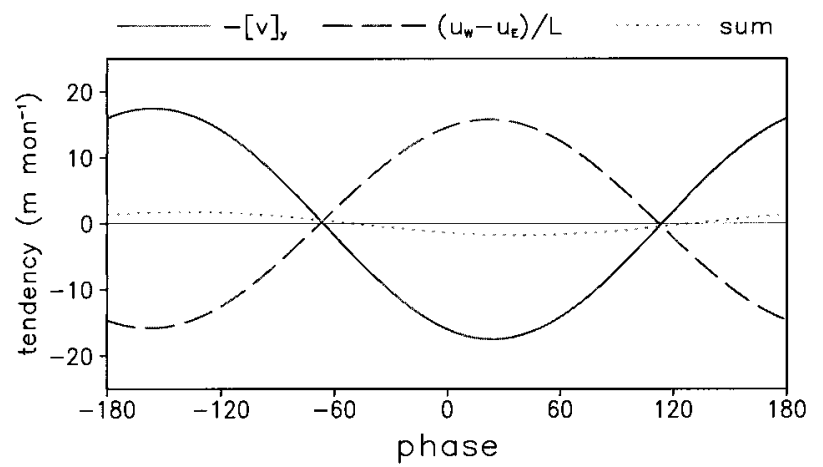

FIG. 3. Variations of the convergence of meridional current $-\partial[v] / \partial y$ and the net zonal flux at the boundaries $\left(u_{W}-u_{E}\right) / L$ and the sum of the two terms for one cycle at the equator. The phase zero indicates the mature phase of warm SST.

where $B$ and $M$ are the net zonal flux at the boundaries and the zonal-mean meridional flux divergence in the ocean interior, respectively. Subscripts 0 and 2, respectively, stand for the components associated with zeroth and second order Hermite polynomials. The derivation of Eqs. (9)-(13) can be found in the appendix. Equations (10)-(13) indicate that the net boundary flux as well as the interior meridional flux divergence are affected by the reflection coefficients.

Figure 4a shows the values of $B_{0}, M_{0}, B_{2}$, and $M_{2}$ at the mature phase of warm SST for different values of $r_{W}$ and a fixed value $\left\{1 /(2)^{1 / 2}\right\}$ of $r_{E}$. In Fig. $4 \mathrm{a}, B_{0}$ is reduced by the increase of $r_{W}$ but $M_{0}$ is not much changed. The reduction of $B_{0}$ with $r_{W}$ can be expected from the equation (10), because the absolute value of $r_{W}-(2)^{1 / 2}$ becomes smaller by the increase of $r_{W}$. The reduction of net boundary flux by the western boundary reflection can be understood by the fact that the zonal current associated with the Kelvin wave reflected at the western boundary has a different sign from that of the Rossby wave, which provides the Kelvin wave by the reflection. For $r_{W}$ less than $0.4, B_{0}$ is bigger than $M_{0}$. For such a small reflection, the warm SST produces a positive tendency of $h_{0}$ and makes further warming near the equator. A reversed situation is true for the cold phase. Thus, in this case the coupled system is unstable without oscillation. On the other hand, for a reflection coefficient bigger than 0.4 , the reflection reduces the boundary zonal flux more than enough to make the tendency of $h_{0}$ toward the other phase of SST.

As expected from Eqs. (12) and (13), $B_{2}$ and $M_{2}$ are not very sensitive to the change of the reflection coefficient at the western boundary (Fig. 4a). Both values are negative for a warm phase. But absolute magnitude of $B_{2}$ is larger than that of $M_{2}$ regardless of $r_{W}$, indicating that the zonal-mean thermocline depth off the equator is determined by the boundary zonal fluxes off the equator rather than the meridional flux convergence. The sensitivity of each component of $\mathrm{h}$ tendency for different value of $r_{E}$ is shown in Fig. $4 \mathrm{~b}$. The absolute magnitudes
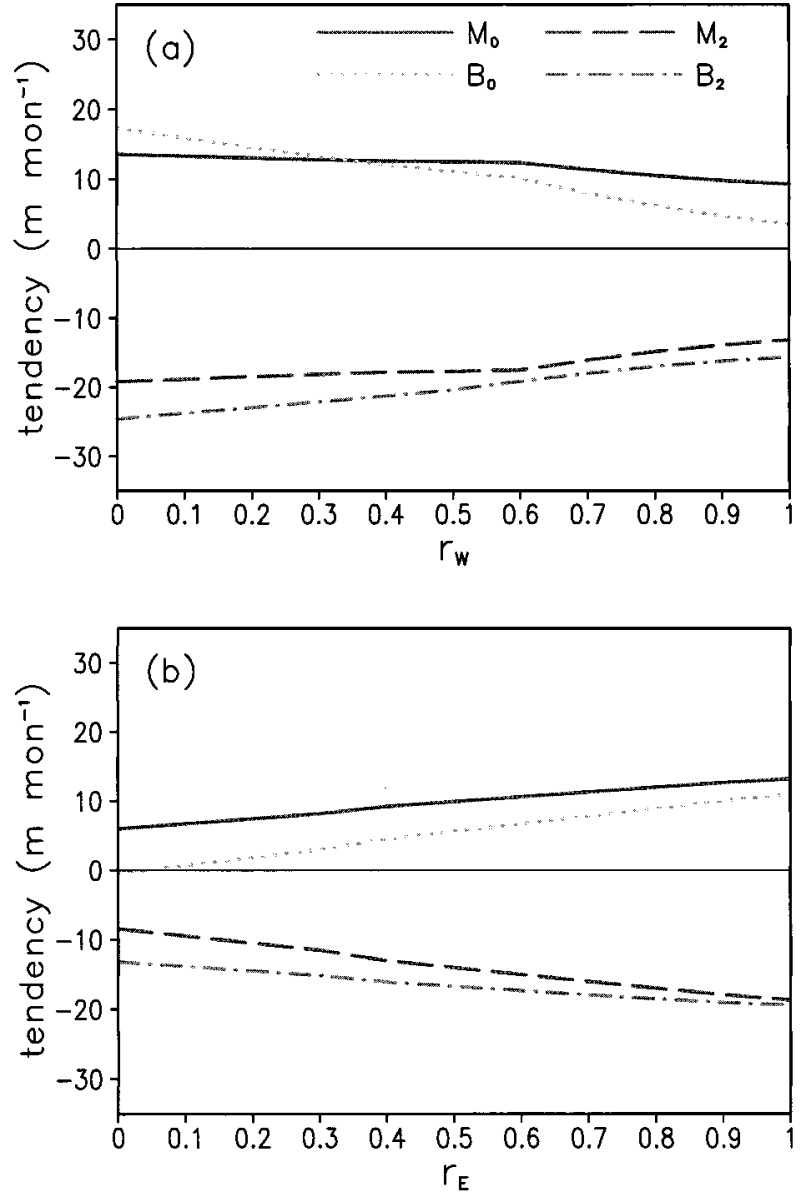

FIG. 4. Dependence of each meridional component of the net zonal flux at the boundaries $(B)$ and the divergence of zonal-mean meridional current $(M)$ on the value of reflection coefficients at the boundaries: (a) and (b) show the western and eastern boundaries, respectively. Plotted are the meridional components of the zeroth- and second-order Hermite polynomials represented by the subscripts of 0 and 2 .

of $M_{0}, M_{2}, B_{0}$, and $B_{2}$ are all increased by the increase of eastern boundary reflection. This is mainly because the east-west difference of $S_{2}, r_{E} S_{0}\left(x_{E}\right)-S_{2}\left(x_{W}\right)$, should be increased by the increase of $r_{E}$. For a given value of $r_{W}=1 /(2)^{1 / 2}$, both $B_{0}-M_{0}$ and $B_{2}-M_{2}$ are negative regardless of $r_{E}$ for a warm phase but positive for a cold phase.

Now we examine the relative importance of the net boundary flux versus the meridional flux convergence for the $h$ tendency at the equator. Figure 5 shows the ratio of the net boundary flux to the meridional flux divergence for different values of the reflection coefficients at the western and eastern boundaries. In obtaining the figure, the reflection coefficient of $1 /(2)^{1 / 2}$ is fixed at the other end of the boundaries. As expected from the previous figure, the ratio is less than 1 for a small value of $r_{W}$. But it is larger than 1 for $r_{W}$ greater than 0.53 and for all values of $r_{E}$. Therefore, the present system can oscillate only when $r_{W}$ exceeds the critical 


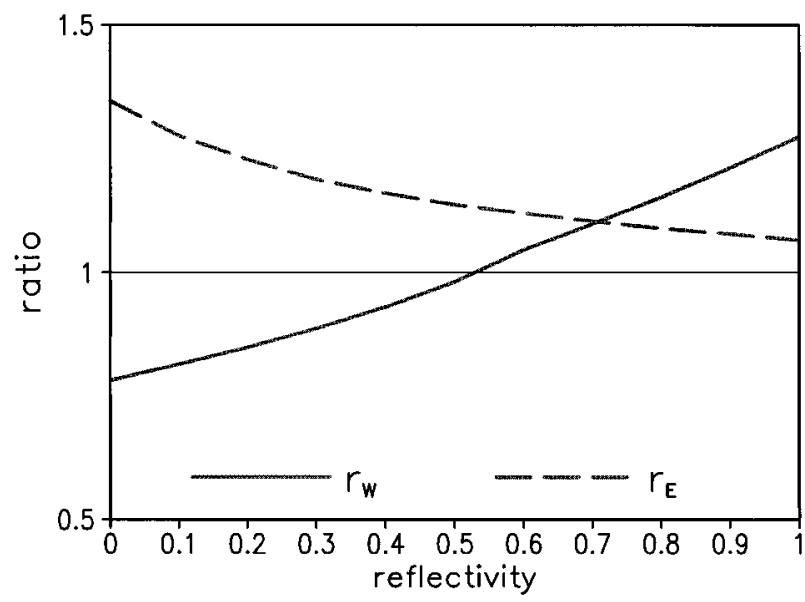

FIG. 5. Ratio of the net boundary flux to the divergence of meridional current for different values of reflection coefficients at the western and eastern boundaries.

value. Kang and An (1998) showed that the present system is unstable without oscillation for the reflection coefficients at the western boundary less than 0.5 but has an oscillatory unstable mode for the coefficient bigger than 0.5. In the present model, both $r_{W}$ and $r_{E}$ are $1 /(2)^{1 / 2}$, and therefore the meridional flux divergence contributes more to the $h$ tendency at the equator than the net zonal boundary flux does.

\section{Conclusions}

The recharge oscillator paradigm suggested by Jin (1997a,b) is further investigated by using a simple coupled model with the zonal mean and eddy parts separated. It is clearly demonstrated that the basin-wide adjustment to the wind stress is accomplished by the net transport of equatorial water off the equator, which is induced by the meridional transport over the ocean interior and the net zonal flux at the boundaries. With a reasonable choice of the reflection coefficients, the meridional transport plays a bigger role and determines the sign of zonal-mean $h$ tendency, in a way that the discharge of equatorial heat content in the warm phase and recharge in the cold phase serve as a phase transition mechanism of the coupled system. In addition, the meridional transport is induced mainly by the geostrophic current associated with the east-west slope of thermocline depth, established quickly by the SST and wind forcing.

In the present system, the negative feedback mechanism played by the meridional transport is accomplished directly by the Rossby waves in the ocean interior. On the other hand, in the delay oscillator, the negative feedback is accomplished by the Rossby wave indirectly with a time delay. The reflection, particularly at the western Pacific, plays a crucial role as a transition mechanism in the delay oscillator. But the role of the reflection is explained differently in the present study, in which the reflection affects the equatorial heat content by modifying the zonal fluxes, particularly at the western boundary. The net boundary flux, which acts as a positive feedback mechanism of the present system, is reduced by the increase of reflection at the western boundary, because at the boundary the zonal current associated with the reflected Kelvin wave has a different sign from that of a forced Rossby wave. The reduction of net boundary flux by the boundary reflection ensures the interior meridional divergence to control the tendency of equatorial heat content. Note that a sufficient reflection at the western boundary ( $r_{W}$ bigger than 0.53 ) is required to make the unstable mode of the present system oscillate.

Acknowledgments. The authors thank Profs. Bin Wang and Fei-Fei Jin for their special interest in this work and thoughtful discussions about the basin-wide adjustment processes. We also appreciate Profs. David Battisti and Ping Chang for their helpful discussions on an early part of the present work. Thanks are extended to Diane Henderson for her careful edit of the manuscript. The first author was supported by the Center for Basic Science in Seoul National University and by the Korea Research Foundation post-doctoral program, and the second author by the Brain Korea 21 Project.

\section{APPENDIX}

\section{Heat Content Exchange at the Equator}

From the equation $u=(S-R) / 2$, the zonal current truncated with the zeroth- and second-order Hermite functions can be expressed as

$u(x, y)=\frac{1}{2}\left\{S_{0}(x)-\sqrt{2} S_{2}(x)\right\} \Psi_{0}(y)+\frac{1}{2} S_{2}(x) \Psi_{2}(y)$.

Using the above equation and the relationships of $S_{0}\left(x_{W}\right)$ $=r_{W} S_{2}\left(x_{W}\right)$ and $S_{2}\left(x_{E}\right)=r_{E} S_{0}\left(x_{E}\right)$, the net convergence of zonal momentum at the boundaries can be written as

$$
\begin{aligned}
\frac{u_{E}-u_{W}}{L}=\frac{1}{2 L}\langle & \left\{S_{0}\left(x_{E}\right)-\sqrt{2} r_{E} S_{0}\left(x_{E}\right)\right\} \Psi_{0}+r_{E} S_{0}\left(x_{E}\right) \Psi_{2} \\
& -\left\{r_{W} S_{2}\left(x_{W}\right)-\sqrt{2} S_{2}\left(x_{W}\right)\right\} \Psi_{0} \\
& \left.-S_{2}\left(x_{W}\right) \Psi_{2}\right\rangle .
\end{aligned}
$$

The meridional convergence of meridional current is written as after using the recurrence formula of Hermite function:

$$
\begin{aligned}
\frac{\partial[v]}{\partial y} & =\frac{\partial}{\partial y}\left(\left[v_{1}\right] \Psi_{1}\right) \\
& =\left\{\frac{2}{3} \frac{r_{E} S_{0}\left(x_{E}\right)-S_{2}\left(x_{W}\right)}{L}-\frac{\sqrt{2}}{6}\left[\tau_{0}\right]\right\}\left\{\frac{\sqrt{2}}{2} \Psi_{0}-\Psi_{2}\right\} .
\end{aligned}
$$


Substituting Eqs. (A2) and (A3) into Eq. (7) after neglecting the damping term and using the orthogonal constraint of $\Psi_{0}$ and $\Psi_{2}$, we can obtain Eqs. (9)-(13).

\section{REFERENCES}

Battisti, D. S., and A. C. Hirst, 1989: Interannual variability in a tropical atmosphere-ocean model: Influence of the basic state, ocean geometry, and nonlinearity. J. Atmos. Sci., 46, 1687-1712.

Bjerknes, J., 1969: Atmospheric teleconnections from the equatorial Pacific. Mon. Wea. Rev., 97, 163-172.

Cane, M. A., and E. S. Sarachik, 1981: The response of a linear baroclinic equatorial ocean to periodic forcing. J. Mar. Res., 39, 651-693.

Chao, Y., and S. G. H. Philander, 1993: On the structure of the Southern Oscillation. J. Climate, 6, 450-469.

Hirst, A. C., 1988: Slow instabilities in tropical ocean basin-global atmosphere models. J. Atmos. Sci., 45, 830-852.
Jin, F.-F., 1997a: An equatorial ocean recharge paradigm for ENSO. Part I: Conceptual model. J. Atmos. Sci., 54, 811-829.

_ 1997b: An equatorial ocean recharge paradigm for ENSO. Part II: A stripped-down coupled model. J. Atmos. Sci., 54, 830-847.

Kang, I.-S., and S.-I. An, 1998: Kelvin and Rossby wave contributions to the SST oscillation of ENSO. J. Climate, 11, 2461-2469.

Li, T., 1997: On the phase transition of the El Niño-Southern Oscillation: A stationary SST mode. J. Atmos. Sci., 54, 2872-2887.

Philander, S. G. H., T. Yamagata, and R. C. Pacanowski, 1984: Unstable air-sea interaction in the Tropics. J. Atmos. Sci., 41, 604613.

Schneider, E. K., B. Huang, and J. Shukla, 1995: Ocean wave dynamics and El Niño. J. Climate, 8, 2415-2439.

Suarez, M. J., and P. S. Schopf, 1988: A delayed action oscillator for ENSO. J. Atmos. Sci., 45, 3283-3287.

Wang, B., and Z. Fang, 1996: Chaotic oscillation of tropical climate: A dynamic system theory for ENSO. J. Atmos. Sci., 53, 27862802.

Wyrtki, K., 1985: Water displacements in the Pacific and the genesis of El Niño cycle. J. Geophys. Res., 91, 7129-7132.

Zebiak, S. E., and M. A. Cane, 1987: A model El Niño-Southern Oscillation. Mon. Wea. Rev., 115, 2262-2278. 